\title{
Using the Stark effect to understand charge generation in organic solar cells
}

\author{
Jelissa De Jonghe-Risse ${ }^{\mathrm{a}}$, Martina Causa ${ }^{\mathrm{b}}$, Ester Buchaca-Domingo ${ }^{\mathrm{c}}$, Martin Heeney ${ }^{\mathrm{c}}$, Jacques-E. \\ Moser $^{\mathrm{a}}$, Natalie Stingelin ${ }^{\mathrm{c}}$, and Natalie Banerji ${ }^{\mathrm{a}, \mathrm{b}}$ * \\ ${ }^{a}$ Institute of Chemical Sciences \& Engineering, Ecole Polytechnique Fédérale de Lausanne (EPFL), \\ SB ISIC GR-MO, Station 6, CH-1015 Lausanne, Switzerland; ${ }^{\mathrm{b}}$ Department of Chemistry, \\ University of Fribourg, Chemin du Musée 9, CH-1700, Switzerland; ${ }^{\mathrm{c}}$ Centre for Plastic Electronics, \\ Imperial College London, Exhibition Road, London, SW7 2AZ, United Kingdom.
}

\begin{abstract}
We have used a femtosecond-resolved spectroscopic technique based on the Stark effect (electromodulated differential absorption) in order to investigate free charge generation and charge drift in solar cell devices of neat conjugated polymer pBTTT and in its 1:1 (by weight) blend with PCBM. In the latter, the fullerene molecules intercalate between the polymer side-chains, yielding a co-crystal phase. Our results show that free charge generation in both materials is ultrafast and strongly dependent on the applied reverse bias. Charge drift to the electrodes (under strong reverse bias) occurs with comparable dynamics on the $1.2 \mathrm{~ns}$ time scale for neat pBTTT and the blend, and is probably dominated by hole transport within/between polymer chains.
\end{abstract}

Keywords: Organic solar cells, charge generation, charge transport, ultrafast spectroscopy, Stark effect, electromodulated differential absorption.

\section{INTRODUCTION}

We have recently shown that charge generation in organic solar cells is strongly correlated to the structure and phase morphology of the active layer medium, i.e. on how the electron donor (typically a conjugated polymer) and the electron acceptor (typically a fullerene derivative) arrange from the nano- to the microscopic scale [1-3]. A particularly interesting model system is pBTTT (poly(2,5-bis(3-hexadecyl-thiophen-2-yl)thieno $[3,2-b]$ thiophene) blended with PCBM ([6,6]-phenyl $\mathrm{C}_{60}$ butyric acid methyl ester), since the phase morphology can in this case be carefully controlled during solution processing $[4,5]$. We present here a relatively new technique, time-resolved electromodulated differential absorption (EDA) [6-9], applied to solar cell devices containing either neat pBTTT or a 1:1 (by weight) blend of pBTTT with PCBM. In neat pBTTT, the polymer chains arrange into ordered lamellae, allowing high transistor mobility [10]. In the presence of PCBM, the fullerene molecules intercalate between the polymer side-chains, yielding a well-defined cocrystal phase [5]. For EDA spectroscopy, a reverse bias was applied to the solar cells and the absorption spectra were measured with and without this external field, resulting in the differential electro-absorption (EA) spectrum. The origin of the EA is the Stark effect, which is the shift of a material's energy levels in an electric field. The samples where then excited with a femtosecond pump pulse, leading to charge generation in the solar cells. When the charges were transported to the electrodes, they shielded the externally applied field, causing a time-dependent reduction of the EA signal. The drift of free charge carriers to the electrodes could thus be followed with femtosecond time resolution.

*natalie.banerji@unifr.ch; http://www.chem.unifr.ch/banerji

Physical Chemistry of Interfaces and Nanomaterials XIV, edited by Sophia C. Hayes, Eric R. Bittner, Proc. of SPIE Vol. 9549, 95490J - (C) 2015 SPIE · CCC code: 0277-786X/15/\$18 - doi: 10.1117/12.2190212 


\section{METHODOLOGY}

The pBTTT polymer $(\mathrm{Mn}=34 \mathrm{kDa} ; \mathrm{Mw}=66 \mathrm{kDa})$ was synthesized as previously reported [10], while PCBM for the blend was purchased from Solenne and used without further purification. Solutions of pBTTT and pBTTT:PCBM (1:1 by weight) were prepared in 1,2-ortho-dichlorobenzene (polymer concentration of 10 $\mathrm{mg} / \mathrm{mL}$ ). All solutions were left stirring for more than 4 hours at $100{ }^{\circ} \mathrm{C}$ to fully dissolve the active material. Thin films were then deposited onto patterned ITO substrates by wire-bar coating (from hot solutions at 85-90 ${ }^{\circ} \mathrm{C}$ onto substrates kept at $35^{\circ} \mathrm{C}$ ), and finally aluminum counter electrodes were thermally evaporated on top of the active layer. Oxygen was removed by keeping the samples for 24 hours in vacuum. They were then sealed with an epoxy resin and a thin glass slide inside the glovebox. The film thickness was approximately 100-110 nm. The steady-state absorption was measured between the Al electrodes with a PerkinElmer Lambda 950 spectrophotometer.

Steady-state EA spectra were recorded on the full solar cell devices with a femtosecond probe beam (white light continuum 400-750 nm), generated by passing a portion of $778 \mathrm{~nm}$ amplified Ti:sapphire output (CPA2001, Clark-MXR, $1 \mathrm{kHz}$ repetition rate) through a $3 \mathrm{~mm}$ sapphire disc. The probe beam entered the sample through the ITO, then reached the active layer and was reflected off the aluminum electrode into the detector. Part of the probe beam was also split before the sample to a reference beam reaching a second detector. The probe and reference were recorded shot-by-shot with a pair of $163 \mathrm{~mm}$ spectrographs (Andor Technology, SR163) equipped with a $512 \times 58$ pixels back-thinned CCD (Hamamatsu S07030-0906) and assembled by Entwicklungsbüro Stresing, Berlin. An electric field (modulated at half the probe pulse frequency) was applied across the solar cell with a function generator (Tektronix AFG 2021). Thus, a square voltage pulse (up to $6 \mathrm{~V}$ in reverse bias) of $100 \mu \mathrm{s}$ duration was applied at $500 \mathrm{~Hz}$, and the reflected probe light was measured shot-by-shot in the presence and in the absence of electric field, averaged over 3000 shots. The final EA spectrum was computed by subtracting the absorption spectra with and without field, correcting for shotto-shot fluctuations using the reference.

For the time-resolved EDA measurements, the devices were excited at $540 \mathrm{~nm}$ with femtosecond pump pulses at a repetition rate of $1 \mathrm{kHz}$. This pump beam was generated with a commercial two-stage non-collinear optical parametric amplifier (NOPA-Clark, MXR) from the $778 \mathrm{~nm}$ laser output. Compression of the NOPA output with two prisms lead to 50-60 fs pulse duration. The pump energy at the sample was adjusted within the hundreds of nanojoule range and the beam diameter was $3.45 \mathrm{~mm}$ (determined with a BC106-Vis Thorlabs beam profiler, $1 / \mathrm{e}^{2}$ cut-off), so that it covered the entire $5 \times 2 \mathrm{~mm}$ active area of the device. The probe intensity was always less than the pump intensity $(<10 \mathrm{~nJ}$ ) and the spot size was much smaller (about 0.2 $\mathrm{mm}$ ). In order to obtain the time-evolution of the EA spectrum after excitation, the probe pulses were time delayed with respect to the pump pulses using a computerized translation stage. The polarization of the probe pulses was at magic angle relative to the pump. In parallel to the optical measurements, the current across the devices was recorded via a $50 \Omega$ series resistor with an oscilloscope (Tektronix TDS 3044B).

\section{RESULTS AND DISCUSSION}

The steady-state absorption spectra, steady-state EA spectra (without sample excitation), and the fielddependence of the EA signature at $620 \mathrm{~nm}$ are shown in Figure 1, for devices containing neat pBTTT or pBTTT:PCBM (1:1 by weight). In the latter, there was full intercalation of the PCBM molecules between the polymer side chains, yielding a one-phase morphology $[4,5]$. Likely due to different molecular order and chain conformation of the polymer macromolecules, the absorption spectra of the neat and blended films were

different, displaying a more pronounced vibronic progression in the case of pBTTT:PCBM [11]. The 
oscillatory EA spectra resembled the first derivative of the corresponding absorption spectra, as expected for a Stark effect caused by a change in polarizability between the ground and excited states [12]. Although the absorbance was higher for the neat pBTTT sample, the electro-absorption was significantly reduced compared to pBTTT:PCBM (Figure 1). This was in part related to the broader, less structured absorption band of the neat polymer (less steep edges lead to a smaller derivative), but also confirmed the different electronic properties of pBTTT in the absence and presence of intercalated PCBM. For both devices, the amplitude of the EA signal recorded at $620 \mathrm{~nm}$ varied quadratically with the applied bias (quadratic Stark effect).

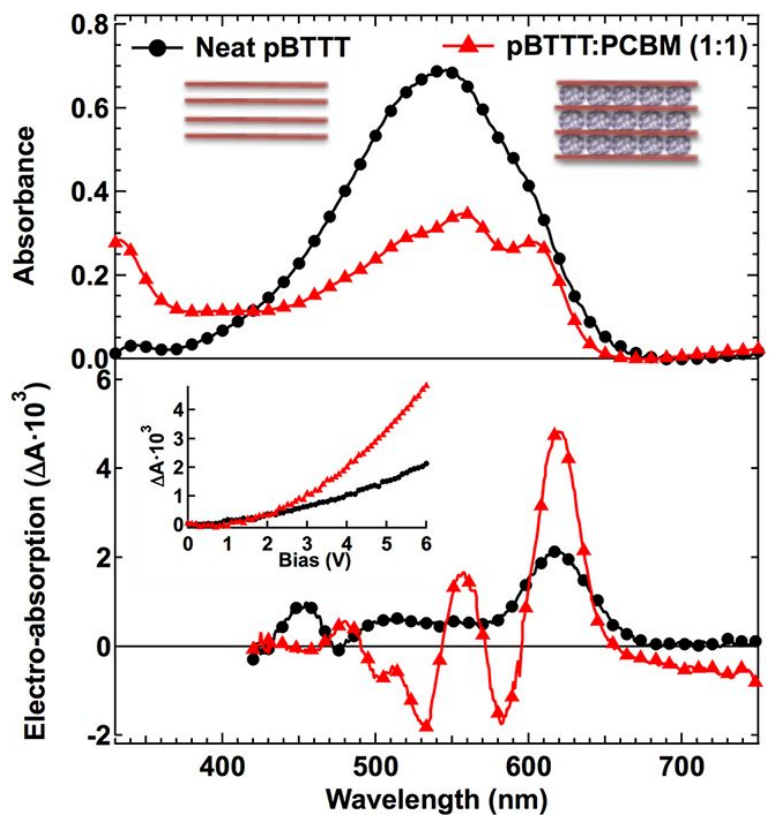

Figure 1. Steady-state absorption spectra of neat pBTTT film and pBTTT:PCBM blend (1:1 by weight), as measured on the used solar cell devices between electrodes (top panel). The insets schematically represent the ordering of the polymer chains, with and without intercalated fullerene molecules. Electro-absorption (EA) spectra recorded for the two samples with an externally applied bias of 6V (bottom panel). The inset shows the bias-dependence of the EA peak amplitude at $620 \mathrm{~nm}$.

The current across the solar cells was recorded with an oscilloscope. When the square voltage pulse (100 $\mu$ s duration) was switched on and off, a current response related to the accumulation/de-accumutation of charges at the device electrodes was observed (inset of Figure 2). Indeed, the devices acted as capacitors in the used range of reverse biases, with minimal charge injection. The applied voltage pulse therefore established a field of the order of $10^{5} \mathrm{~V} / \mathrm{cm}$ across the active layer. When the devices were excited by the $540 \mathrm{~nm}$ pump pulse during the application of the external reverse bias, an additional photocurrent transient appeared on the oscilloscope. Its integral (after subtraction of the dark current) yielded the extracted photo-generated charge. In Figure 2, the extracted charge as a function of applied bias (at a fixed pump fluence of $5.3 \mu \mathrm{J} / \mathrm{cm}^{2}$ ), and as a function of pump fluence (at a fixed reverse bias of $2.5 \mathrm{~V}$ ) are shown. The extracted charge was generally much lower in the neat pBTTT device compared to the pBTTT:PCBM blend, in agreement with low photocurrent yield for conjugated polymer films in the absence of an electron acceptor [13]. Nevertheless, the extracted charge increased significantly with applied bias, for both the pBTTT and pBTTT:PCBM cells. This implies a strong field-dependence of charge generation and/or charge extraction in both materials. In pBTTT:PCBM (1:1 by weight), it has been shown that the intimate contact of the donor and acceptor in the co-crystal phase lead to highly efficient, ultrafast charge separation [1]. In the absence of an external field, 
most electron-hole pairs nevertheless geminately recombined instead of spatially separating. The increased yield of extracted charge with reverse bias observed here can be explained by the concept that the application of the external bias helped the electron-hole pairs to overcome their mutual Coulomb attraction, thus preventing geminate recombination [14].

At an applied reverse bias of $2.5 \mathrm{~V}$, the extracted charge in the pBTTT:PCBM blend increased linearly up to a pump fluence of only $2 \mu \mathrm{J} / \mathrm{cm}^{2}$ (Figure 2), then this relation became sub-linear, confirming the presence of a significant concentration of free charge carriers (as opposed to bound electron-hole pairs) undergoing bimolecular recombination. In contrast, the extracted charge in neat pBTTT varied linearly with pump fluence over a much more important range (the free charge concentration remained too low for bimolecular effects).
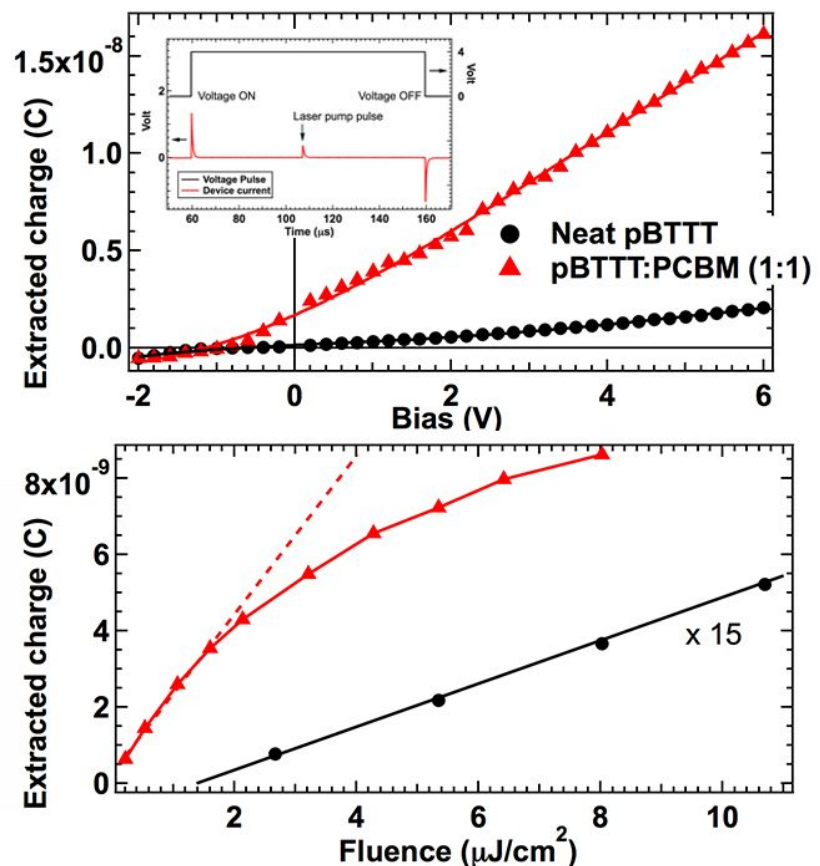

Figure 2. Photo-generated charge extracted from the neat pBTTT and pBTTT:PCBM (1:1 by weight) devices, obtained by integration of the photocurrent transients measured via a $50 \Omega$ series resistor with an oscilloscope after subtraction of the dark current, as a function of applied reverse bias (top panel, at a fluence of $5.3 \mu \mathrm{J} / \mathrm{cm}^{2}$ ), and as a function of fluence (bottom panel, at an applied reverse bias of $2.5 \mathrm{~V}$ ). The inset shows the typical current response measured over the device, upon switching on/off the voltage pulse and upon irradiating with the $540 \mathrm{~nm}$ pump pulse.

The drift of the free charge carriers toward the electrodes opposed the external reverse bias and thus reduced the field within the active layer. This was directly observed with femtosecond resolution as a decrease of the EA signal at different time delays after photo-excitation. Using the quadratic relation between the EA amplitude at $620 \mathrm{~nm}$ and the applied voltage (inset of Figure 1), the decrease of the EA could be directly translated to a voltage drop across the solar cells (Figure 3). At the same pump fluence of $1.6 \mu \mathrm{J} / \mathrm{cm}^{2}$, the total voltage drop at $1.2 \mathrm{~ns}$ after excitation was $<0.1 \mathrm{~V}$ in neat pBTTT, while it was around $0.6 \mathrm{~V}$ in the pBTTT:PCBM blend. This confirmed the much higher efficiency of free charge generation in presence of the fullerene, even at early time scales after excitation. The voltage drop in the neat pBTTT device could be significantly increased at higher pump fluence $\left(5.3 \mu \mathrm{J} / \mathrm{cm}^{2}\right)$, in agreement with the linear increase of extracted charge with pump intensity. It now became clear that the decay of the electric field due to charge drift 
followed similar dynamics in the neat sample as in the blend (Figure 3). Those dynamics were strongly multiphasic, with time constants ranging from 2 ps to $600 \mathrm{ps}$ (dispersive transport). The immediate onset of the voltage drop after photo-excitation in both materials pointed to ultrafast generation of the free charge carriers. In the neat sample, this might occur via hot exciton dissociation [15]. The ensuing comparable EDA dynamics indicated that the carrier transport under strong reverse bias on the $1.2 \mathrm{~ns}$ time scale occurred by a comparable mechanism in the neat and blended device, most probably dominated by hole transport within/between the polymer chains.

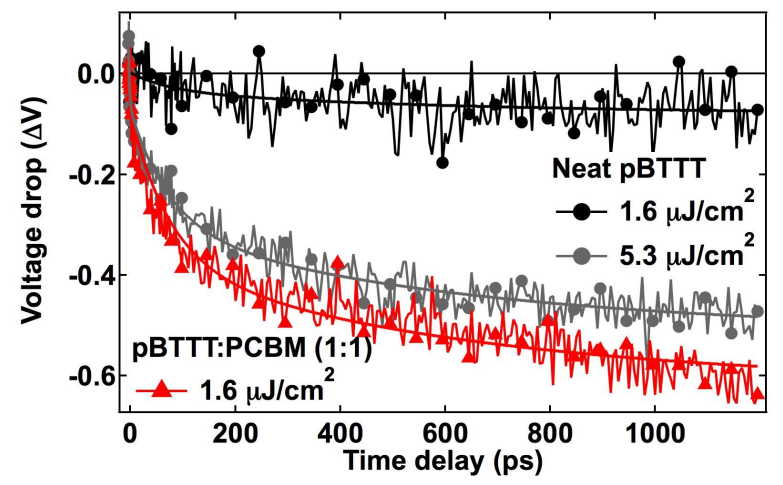

Figure 3. Voltage drop across the pBTTT and pBTTT:PCBM devices excited at $540 \mathrm{~nm}$, as obtained from the femtosecond-resolved decay of the EA signal at $620 \mathrm{~nm}(6 \mathrm{~V}$ external reverse bias).

\section{CONCLUSION}

We have shown that femtosecond-resolved electromodulated differential absorption (EDA) spectroscopy is a useful tool to investigate free charge generation and charge drift in solar cell devices. For samples of neat pBTTT and in its 1:1 (by weight) blend with PCBM, we could evidence ultrafast, highly field-dependent generation of free charges, which was much more efficient in the presence of the fullerene. Charge drift to the electrodes on the $1.2 \mathrm{~ns}$ time scale occurred with comparable dynamics under strong reverse bias.

[1] M. Scarongella, J. De Jonghe-Risse, E. Buchaca-Domingo et al., "A close look at charge generation in polymer:fullerene blends with microstructure control," J. Am. Chem. Soc., 137(8), 2908-2918 (2015).

[2] M. Scarongella, A. A. Paraecattil, E. Buchaca-Domingo et al., "The influence of microstructure on charge separation dynamics in organic bulk heterojunction materials for solar cell applications," J. Mater. Chem. A, 2(17), 6218-6230 (2014).

[3] A. A. Paraecattil, and N. Banerji, "Charge separation pathways in a highly efficient polymer: fullerene solar cell material," J. Am. Chem. Soc., 136(4), 1472-1482 (2014).

[4] E. Buchaca-Domingo, A. J. Ferguson, F. C. Jamieson et al., "Additive-assisted supramolecular manipulation of polymer:fullerene blend phase morphologies and its influence on photophysical processes," Mater. Horiz., 1(2), 270-279 (2014).

[5] A. C. Mayer, M. F. Toney, S. R. Scully et al., "Bimolecular crystals of fullerenes in conjugated polymers and the implications of molecular mixing for solar cells," Adv. Funct. Mater., 19(8), 11731179 (2009). 
[6] J. Cabanillas-Gonzalez, T. Virgili, A. Gambetta et al., "Photoinduced transient stark spectroscopy in organic semiconductors: A method for charge mobility determination in the picosecond regime," Phys. Rev. Lett., 96(10), 106601 (2006).

[7] A. Devizis, J. De Jonghe-Risse, R. Hany et al., "Dissociation of charge transfer states and carrier separation in bilayer organic solar cells: A time-resolved electroabsorption spectroscopy study," J. Am. Chem. Soc., 137(25), 8192-8198 (2015).

[8] A. Devižis, D. Hertel, K. Meerholz et al., "Time-independent, high electron mobility in thin $\mathrm{PC}_{61} \mathrm{BM}$ films: Relevance to organic photovoltaics," Org. Electron., 15(12), 3729-3734 (2014).

[9] V. Gulbinas, R. Kananavicius, L. Valkunas et al., "Dynamic Stark effect as a probe of the evolution of geminate electron-hole pairs in a conjugated polymer," Phys Rev B, 66(23), 233203 (2002).

[10] I. Mcculloch, M. Heeney, C. Bailey et al., "Liquid-crystalline semiconducting polymers with high charge-carrier mobility," Nat. Mater., 5(4), 328-333 (2006).

[11] N. C. Miller, E. Cho, M. J. Junk et al., "Use of X-ray diffraction, molecular simulations, and spectroscopy to determine the molecular packing in a polymer-fullerene bimolecular crystal," Adv. Mater., 24(45), 6071-6079 (2012).

[12] G. Lanzani, [The photophysics behind photovoltaics and photonics] Wiley-VCH Verlag\&Co Weinheim (2012).

[13] O. G. Reid, and G. Rumbles, "Quantitative transient absorption measurements of polaron yield and absorption coefficient in neat conjugated polymers," J. Phys. Chem. Lett., 4(14), 2348-2355 (2013).

[14] A. Zusan, K. Vandewal, B. Allendorf et al., "The crucial influence of fullerene phases on photogeneration in organic bulk heterojunction solar cells," Adv. Energy Mater., 4(17), 1400922 (2014).

[15] V. I. Arkhipov, E. V. Emelianova, S. Barth et al., "Ultrafast on-chain dissociation of hot excitons in conjugated polymers," Phys Rev B, 61(12), 8207-8214 (2000). 